\title{
UPDATING LAND COVER DATABASES USING A SINGLE VERY HIGH RESOLUTION SATELLITE IMAGE
}

\author{
Adrien Gressin ${ }^{1,2}$, Clément Mallet ${ }^{1}$, Nicole Vincent ${ }^{2}$ and Nicolas Paparoditis ${ }^{1}$ \\ ${ }^{1}$ IGN/SR, MATIS, Université Paris-Est, 73 avenue de Paris, 94160 Saint-Mandé, France; \\ (firstname.lastname@ign.fr), \\ ${ }^{2}$ LIPADE - SIP, Paris-Descartes University, 45 Rue des Saint-Pères, Paris, France; \\ (nicole.vincent@mi.parisdescartes.fr)
}

KEY WORDS: change detection, updating, classification, image, 2D topographic database, very high resolution, satellite.

\begin{abstract}
:
Image change detection has been extensively tackled in the literature in various domains, and in particular for remote sensing purposes. Indeed, very high resolution geospatial images are nowadays ubiquitous and can be used to update existing 2D and 3D geographical databases. Such databases can be projected into the image space, by a rasterization step. Therefore, they provide 2D label maps that can be subsequently compared with classifications resulting for geospatial image processing. In this paper, we propose a classificationbased method to detect changes between label maps created from 2D land-cover databases and an more recent orthoimage, without any prior assumptions about the databases composition. Our supervised method is based both on an efficient training set selection and a hierarchical decision process, that follows the structure of topographical databases. This allows to take into account the intrinsic heterogeneity of the objects and themes composing a database while limiting false detection rates, a standard limitation of existing approaches. The designed framework is successfully applied on very high resolution images of Pléiades sensor and two distinct national land-cover databases.
\end{abstract}

\section{INTRODUCTION}

2D topographic land-cover databases are now available over most of the extent of many developed countries. Such databases are mainly created by merging existing databases of various kinds and sources. Classes of high interest that are not in such databases are derived according to ground surveys, photo-interpretation of very high resolution images, or development of automatic themespecific detection algorithms.

In France, the generation of the new global 2D land-cover/landuse $(\mathrm{LC} / \mathrm{LU})$ database at a spatial resolution of $1 \mathrm{~m}$ has started in 2013, on the initiative of several public institutions. It is organised in a hierarchical manner, similarly to Corine Land Cover, so as to fit with a large number of users' needs. Furthermore, it aims to federate various existing databases both at national and regional levels (buildings, roads, water areas, rivers, forests, agriculture etc.). After the generation of the initial state, two issues remain challenging. First, the completeness and the geometrical accuracy of all the classes included in these databases may vary significantly and one has to detect discrepancies with respect to the database specifications. Secondly, such a database needs to be updated at least every year. Since most classes are the result of human intervention, automatic change detection processes should be set up and be valid for each of these labels.

The aim of this paper is to present a new method to solve both issues equally, using geospatial images at the same resolution as the LC/LU database, namely very high resolution satellite images, without any prior assumption about the database classes. The recent launch of very high resolution (inferior to $1 \mathrm{~m}$ ) satellite sensors, such as Pléiades or Worldview-2, allow to obtain a regular coverage of large areas in a very short period of time. However, despite the ability of such sensors to acquire stereoscopic images, their scheduling and cost issues often limit the disposal to a single image per period (monoscopic configuration). In this paper, we will therefore focus on single images of sub-metric resolution, regularly acquired over the year, to automatically update land-cover databases.

Change detection is a main subject of research in the image processing domain, e.g., for video surveillance, infrastructure mon- itoring or medical diagnostic (Radke et al., 2005; Goyette et al., 2012). In remote sensing, a large body of literature has tackled the problem, from many points of view. For instance, changes can be detected between two images (Miller et al., 2005), or several high or very high resolution images (time-series) (Robin et al., 2010; Petitjean et al., 2012; Bovolo et al., 2013). Hussain et al. (2013) provide a good overview and taxonomy of change detection methods.

More specifically, detecting changes between a LC/LU database (DB) and images more recent than the images or the observations that allowed to populate the DB is a main subject of interest (Holland et al., 2006; Gianinetto, 2008; Champion et al., 2010). For example, Marçal et al. (2005) match objects of a DB with image saliencies, in order to select good training sets for the supervised classification of a multi-spectral image, using Support Vector Machine and Logistic Discrimination methods. Walter (2004) segments aerial images according to DB object outlines and performs an object-based classification using a supervised Maximum Likelihood classification, eventually compared with the initial DB for change detection. More generally, one can notice that existing methods focus on a very specific object type (e.g., roads, buildings), and require the computation of 3D features from stereoscopic images, in order to better discriminate such objects (Nemmour and Chibani, 2006; Poulain et al., 2009; Champion et al., 2010). Helmholz et al. (2012) integrate such specific methods in a global semi-automatic workflow for detecting changes between a 2D geographical DB and orthorectified up-to-date images. Each object of the DB is verified by an automatic image analysis operator, integrated into a knowledge-based image interpretation system. This work is closely related to our issues, but specific methods have to be developed for each kind of label (also called theme) of the DB, and new classes would not be directly supported. Thus, this method cannot be adopted here. Finally, the analysis of the approaches mentioned above allows us to conclude that:

$\triangleright$ A supervised classifier should be adopted: this is the best way to fit to the various kinds of themes of the DB and to the variability of the geographic areas. We can also learn the underlying statistics of the data, instead of applying some rule-based model 
or unsupervised clustering approaches;

$\triangleright$ As few changes exist between the DB and the image, the DB can be kept as initial solution to select reliable training sets for the classifier.

Such assumptions will be the basis of the solution proposed here. Nevertheless, contrary to approaches developed so far, our method targets to detect any change (new, modified, and deleted objects) from any theme of the DB of interest. The robustness of the change detection system will be mainly guaranteed by the selection of the training sets that best discriminate each object of the DB from the rest of the image. Such a per-object consideration will then be propagated into the hierarchical layout of the DB by merging information at each DB level in order to strengthen the process. Our method introduces several new relevant features:

$\triangleright$ No restricting inputs. A single very high resolution $(<1 \mathrm{~m})$ satellite image is required, without stereoscopic configuration. To deal with such a weak input, a very high number of features are computed from the image, and we assume they will be sufficient to learn the visual aspect of the different themes of the DB;

$\triangleright$ Independence to the objects of interest. Our feature set and training step are not designed for specific object types. Therefore, our method can be applied on any 2D geographical database;

$\triangleright$ The use of the hierarchical layout of the DB allows a bottomup analysis (object $\rightarrow$ theme $\rightarrow$ DB) and to obtain LC and change maps consistent with the different objects populating each theme of the DB.

An overview of our change detection methodology is first introduced in Section 2.1. Then, the hierarchical approach is detailed in Section 2.2. Results are presented for two different 2D topographic databases at two different levels of details in Section 3. Finally, conclusions are drawn in Section 4.

\section{METHODOLOGY}

In this section, we first provide an overview of our methodology and introduce some notations. Then, the three levels of our approach are detailed. Finally, technical choices are discussed.

\subsection{Overview}

2.1.1 Inspection principle 2D geographical databases (DB) are structured in three different levels: a DB is composed of nonoverlapping themes, and themes are themselves composed of objects, that are in practice 2D polygons (cf. Figure 1a). Therefore, this hierarchical layout exhibits three levels of possible examination: (1) the object level, (2) the theme level, and (3) the DB level. The initial DB is resampled at a resolution consistent with both the geometrical accuracy of its themes and the spatial resolution of the satellite image. Here, $1 \mathrm{~m}$ is selected as the most appropriate scale of analysis. Since no overlapping themes exist in a DB, a pixel of such a grid can include not more than one label, corresponding to the theme of the object intersecting such pixel. Our methodology is based on such hierarchical inspection, in a "bottom-up" way. Our method first performs a classification per each object of the database. Then, labels are merged at the theme level, and the final decision is taken at the DB level (Figure 1b). The object level inspection consists (1) in choosing, for each object, a subset of pixels that best allows to discriminate the object from the rest of the image (called here outside), and (2) in classifying the full image into two labels (inside / outside). The subset selection is based on the maximization of the recall a two-class classification of the pixels of the object (Section 2.1.2). Thereafter, the labelling process allows to obtain a probability map for each object of each theme of the database.

All classifications computed at the object level are then merged at the theme level, in order to obtain a single probability map per theme. This map describes the probability of each pixel of the image to belong to the current theme. Thanks to the object level inspection, the several existing visual aspects of the theme are kept and no specific ones are favored (e.g., ones of the largest objects).

Finally, a decision is taken at the DB level. A new label image is first produced, that associates to each pixel one label of the initial DB. Secondly, a final probability change map is generated, allowing to label each pixel of the DB image as changed or not changed, and to detect confusion areas. This indicates where our method cannot straightforwardly take a decision. The reduction of those confusing areas will be the purpose of our next research.

\subsubsection{Notations}

Database A 2D geographical database is structured in several themes $\left\{T_{j}\right\}_{j \in\left[1, N_{T}\right]}, N_{T}$ being the number of themes (Equation 1). Each theme $T_{j}$ is itself composed of a set of objects $\left\{O_{i}^{j}\right\}_{i \in\left[1, N_{T_{j}}\right]}, N_{T_{j}}$ being the number of objects of the theme $T_{j}$ (see Figure 1a and Equation 2).

$$
\begin{aligned}
\mathrm{DB} & =\bigcup_{j \in\left[1, N_{T}\right]} T_{j}, \\
\forall j \in\left[1, N_{T}\right], \quad T_{j} & =\bigcup_{i \in\left[1, N_{T_{j}}\right]} O_{i}^{j} .
\end{aligned}
$$

Projection onto the image Each object $O_{i}^{j}$ of the theme $T_{j}$ is associated to a region $R_{i}^{j}$ of the image, by a projection function $\mathcal{I}$, given in Equation 3. Thus, each region $R_{i}^{j}$ is a set of pixels of the image. Thereafter, object $O_{i}^{j}$ and its image projection $R_{i}^{j}$ will be noted equally, and the notation $O_{i}^{j}$ will be used.

$$
\forall j \in\left[1, N_{T}\right], \quad \forall i \in\left[1, N_{T_{j}}\right], \quad R_{i}^{j}=\mathcal{I}\left(O_{i}^{j}\right) .
$$

Moreover, when no ambiguity is possible, an object $O_{i}^{j}$ and its associated theme $T_{j}$ will be noted $O$ and $T$. The projection of all the objects composing a DB may not necessary cover the full area of the region of interest $\Omega$ : the rest of the area is denoted background hereafter:

$$
\Omega=P_{I}(\mathrm{DB}) \cup \text { background } .
$$

Finally, each pixel of the image is initially labelled either by a theme of the DB either by being in the background $\left(\left\{B_{g}\right\}\right)$. The label of a pixel $p$ of the image $I$ is given by the function $L$ as:

$$
\begin{aligned}
L_{\mathrm{DB}}: I & \longrightarrow\left\{T_{j}\right\}_{j \in\left[1 \ldots N_{T}\right]} \cup\{B g\} \\
p & \longmapsto L_{\mathrm{DB}}(p)
\end{aligned}
$$

Classification Changes between the DB and the satellite image are assumed to be limited so that DB can be used to learn the visual aspect of existing objects, and therefore themes. Thus, DB objects are used to train the classifier, introduced in Sec. 2.3. Moreover, the training step is performed according to a subset of pixels $E$, labelled in two classes $\left\{l_{1}, l_{2}\right\}$, with the function $l$ (Equation 4). $\{E, l\}$ is called the training set, and can also be noted $\left(E_{1}, E_{2}\right)$, where $E_{1}=\left\{p \in E, l(p)=l_{1}\right\}$ and $E_{2}=$ $\left\{p \in E, l(p)=l_{2}\right\}$.

$$
\begin{aligned}
l: E & \longrightarrow\left\{l_{1}, l_{2}\right\} \\
p & \longmapsto l(p)
\end{aligned}
$$

From the training set $\{E, l\}$, the classification method allows to define a classification function $l_{c}$, that is applied to the full image:

$$
\begin{aligned}
l_{c}: I & \longrightarrow\left\{l_{1}, l_{2}\right\} \\
p & \longmapsto l_{c}(p)
\end{aligned}
$$

A probability measure is derived from the classification, this measure is defined as the probability of each pixel of the image to belong to the class $l_{1}, P\left(l_{c}(p)=l_{1}\right)$, the probability to belong to $l_{2}$ being equal to $1-P\left(l_{c}(p)=l_{1}\right)$. Finally, a map of probabilities $\mathcal{P}(E, l)=\mathcal{P}\left(E_{1}, E_{2}\right)$ is defined as follows: 


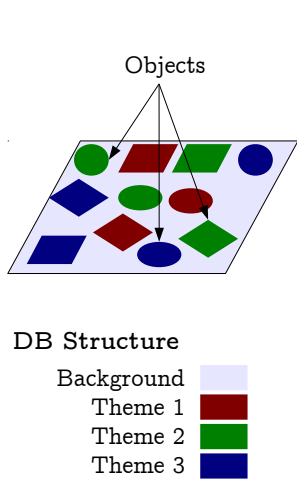

(a)

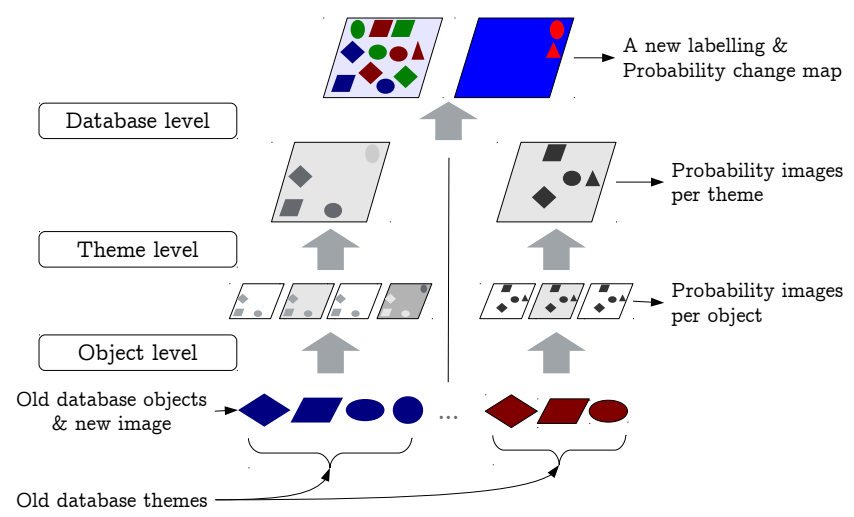

(b)

Figure 1: (a) Database structure and (b) our hierarchical inspection methodology.

$$
\begin{aligned}
\mathcal{P}(E, l)=\mathcal{P}\left(E_{1}, E_{2}\right): I & \longrightarrow[0,1] \\
p & \longmapsto P\left(l_{c}(p)=l_{1}\right)
\end{aligned}
$$

Thereafter, the recall of a classification $l_{c}$, with regard to a groundtruth $G T=\left\{E_{G T}, l_{G T}\right\}$ (a subset of pixels whose label is known a priori), is defined as the number of pixels of $E_{G T}$ that are correctly labelled by $l_{c}$ :

$$
\operatorname{Recall}\left(l_{c}, G T\right)=\operatorname{Card}\left(p \in E_{G T}, l_{c}(p)=l_{G T}(p)\right) .
$$

\subsection{The hierarchical process}

The three levels of our inspection method are detailed thereafter. 2.2.1 Object level The object level inspection is based on a supervised classifier that is fed with a set of image-based features, described in Section 2.3. For each object, a specific classifier is trained on the subset of pixels, taken inside the object, able to best discriminate the object from the rest of the pixels of the image and out of the current theme. The selection of such a training set also allows to reduce registration issues between DB and images that is often reported in the literature (Poulain et al., 2009).

Training set selection The purpose of this part is to chose two pixels sets for appropriate training: one in the object $O$ of the theme $T\left(S_{O}^{i n^{*}} \subset O\right)$, and one out of the current theme $\left(S_{O}^{\text {out }}{ }^{*} \subset\right.$ $\left.I \backslash P_{I}(T)\right)$. $\left\{S_{O}^{\text {in* }}, S_{O}^{\text {out }}\right\}$ defines the full training set $S_{O}^{*}$. These two subsets are selected among several randomly generated subsets, as the subsets that maximise the recall of the classification (theme / out of theme). Such selection is performed in two steps: (1) the selection of the best inside subset, and (2) the selection of the best outside subset.

First, the inside subset is selected as follows: a subset of outside pixels is randomly drawn within pixels out of the current object, $S_{O}^{\text {out }} \subset I \backslash P_{I}(T)$. Those pixels can belong to another theme or to the background. Then, $N_{\text {in }}$ inside pixels are randomly drawn within pixels of the current object:

$$
\left\{S_{O}^{i n_{j}}\right\}_{j \in\left[1 . . N_{i n}\right]} \subset O^{N_{i n}} .
$$

One classification is obtained per each inside subset, paired with the outside one, $\left\{l_{c}^{i n_{j}}\right\}_{j \in\left[1 . . N_{i n}\right]}$. The recall of each classifier is computed by using object pixels as ground-truth, $G T=\left\{P_{I}(O), l\right\}$. The inside subset with the best recall is selected according to:

$$
i n^{*}=\arg \max _{j \in\left[1 . . N_{i n}\right]} \operatorname{Recall}\left(l_{c}^{i n_{j}}, G T\right) \text {. }
$$

Secondly, the outside subset is selected in the same way. $N_{\text {out }}$ outside subsets are randomly drawn, and the corresponding classifiers $\left\{l_{c}^{\text {out }_{j}}\right\}_{j \in\left[1 . . N_{\text {out }}\right]}$ are trained:

$$
\begin{aligned}
& \left\{S_{O}^{\text {out }_{j}}\right\}_{j \in\left[1 . . N_{\text {out }}\right]} \subset\left(I \backslash P_{I}(T)\right)^{N_{\text {out }}},
\end{aligned}
$$

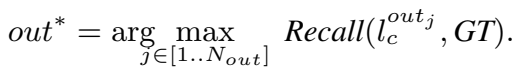

Finally, the training set is defined as the union of the best inside subset $S_{O}^{i n^{*}}$ and the best outside one $S_{O}^{\text {out }}{ }^{*}$.

$$
S_{O}^{*}=S_{O}^{i n^{*}} \cup S_{O}^{\text {out }}{ }^{*} \text {. }
$$

Classification The optimal training subset $S_{O}^{*}$, associated to the object $O$ is then used to perform a classification and to retrieve a probability map $\mathcal{P}_{O}$ :

$$
\mathcal{P}_{O}=\mathcal{P}\left(S_{O}^{*}\right)=\mathcal{P}\left(S_{O}^{i^{*}}, S_{O}^{e^{*}}\right) .
$$

2.2.2 Theme level The probability maps $\mathcal{P}_{O}$, computed for each object $O$ of a given theme $T$, are merged in order to obtain a single probability map per theme $\mathcal{P}^{T}$ :

$$
\mathcal{P}^{T}=\operatorname{Fusion}\left(\left\{\mathcal{P}_{O}, \quad O \in T\right\}\right) .
$$

For now, three merging methods have been tested, the minimal, the maximal and the weighted mean value. In this paper, the mean weighted by the size of the objects is used, in order to give more confidence in the larger objects, that are less likely to change. However, merging probability maps with Bayes rules is planed.

2.2.3 Database level For each pixel, the set of probabilities to belong to each theme $\left\{\mathcal{P}^{T}\right\}_{T \in D B}$ is used to obtain the final label, corresponding to one of the theme, and a confidence map of such a labelling. Then, a final change map is computed by merging (1) the difference between the initial DB and the new classification, and (2) the confidence map.

Labelling and confidence map The final labelling $L_{C_{1}}$ is obtained by keeping, for each pixel, the theme with the highest probability value. It is defined as follows:

$$
\begin{aligned}
L_{C_{1}}: I & \longrightarrow\left[1 . . N_{T}\right] \\
p & \longmapsto \underset{j \in\left[1 . . N_{T}\right]}{\arg \max } \mathcal{P}^{T_{j}}(p)
\end{aligned}
$$

A confidence value $C$ is associated with the classification $L_{C_{1}}$. It allows not to limit the system to a binary decision and to enhance confusion areas, i.e., areas where no straightforward decision can be taken. In practice, the maximal value of the probabilities of each theme provides a reliable measure of the confidence in the previous labelling:

$$
\begin{aligned}
C_{\text {max }}: I & \longrightarrow[0,1] \\
p & \longmapsto \mathcal{P}^{T_{L_{C_{1}}(p)}(p)}
\end{aligned}
$$

However, if two themes have both a high probability value, the maximum value is indeed high but the confidence should be low. Thus, a marging measure is preferred. It is defined as the difference between the two highest probability values, related to $L_{C_{1}}$ and $L_{C_{2}}$ :

$$
\begin{aligned}
C_{\text {margin }}: I & \longrightarrow[0,1] \\
p & \longmapsto \mathcal{P}^{T_{L_{C_{1}}}(p)}(p)-\mathcal{P}^{T_{L_{C}}(p)}(p),
\end{aligned}
$$


where $L_{C_{2}}(p)$ is the theme with the second highest probability value in $p$.

Probability change map A change map $\Delta$ is obtained by comparing new labels with the initial DB. Thus, each pixel can take, either the value -1 (change), either the value 1 (no change):

$$
\forall p \in I, \quad \Delta(p)=\left\{\begin{aligned}
-1 & \text { si } L_{C_{1}}(p) \neq L_{\mathrm{DB}}(p) \\
1 & \text { si } L_{C_{1}}(p)=L_{\mathrm{DB}}(p)
\end{aligned}\right.
$$

This change map $\Delta$ is then weighted by the confidence measure, in order to derive a final probability change map $\Pi$ such as:

$$
\forall p \in I, \quad \Pi(p)=\Delta(p) * C(p) .
$$

This map is composed of three classes: certain change, certain no-change, and uncertain (i.e., confusion areas, see Equation 6). For that purpose, two thresholds $S_{1}$ and $S_{2}$ are introduced. They should be tuned according to the quality result and the percentage of false alarms that is tolerated.

$$
\forall p \in I, \begin{cases}\text { certain change } & \text { if } \Pi(p) \leq S_{1} \\ \text { certain no-change } & \text { if } \Pi(p) \geq S_{2} \\ \text { uncertain } & \text { otherwise }\end{cases}
$$

Such labelling allows to focus an operator either on change areas, either on confusion areas.

\subsection{Technical choices}

2.3.1 Classifier The classification method must be adapted to the different themes of the analysed DB. Thus, a supervised classification is chosen. Moreover, such a method should (1) handle a very large numbers of features $(>200)$, (2) have a high generalisation ability, and (3) be fast enough to perform as many classifications as required. Consequently, a Support Vector Machine (SVM) method was chosen (Foody et al., 2006), with a standard Gaussian kernel $\left(k\left(x_{1}, x_{2}\right)=C * e^{-\gamma\left\|x_{1}-x_{2}\right\|^{2}}\right.$, where $\left(x_{1}, x_{2}\right)$ are two feature vector). A grid search method was applied to select the best hyper-parameters $(\mathrm{C}, \gamma)$ that maximise the crossvalidation accuracy. Each SVM provides a binary classification, coupled with a probability estimate per theme (Wu et al., 2004).

2.3.2 Features Our methodology is based on the fact that the various visual aspects of each theme of the DB can be retrieved. Since the most relevant features for a specific theme cannot be known beforehand, and since we aim to deal with any kind of theme, a large number of features computed from the different channels of the image is therefore required. The quality of our workflow is highly dependent on those features. A large body of literature has dealt with geospatial image-based feature extraction (Lefebvre et al., 2008; Lienou and Campedel, 2009; Tokarczyk et al., 2012). Features can be (1) spectral (resulting from the combination of the input spectral channels of the image), (2) geometrical (i.e., low-level primitives such as lines or keypoints), and (3) textural (describing the image behaviour in the neighbourhood of each pixel).

With a sub-metric image, a multi-scale observation may be useful. Indeed, a small neighbourhood will probably provide more homogeneous textures and best describes fine textures (e.g., forest or fields), while large neighbourhood allows to better estimate complex textures as vineyard or aligned buildings, (Campedel et al., 2005).

\subsection{Training point selection}

The number of training points is tuned to 200 (100 inside and 100 outside), and the numbers of random selections $N_{\text {in }}$ and $N_{\text {out }}$ is set to 10 . However, a quantitative study will be carried out to know the real sensitiveness of such parameters with respect to the themes, and the area of interest.

\section{RESULTS ON LAND COVER DATASETS}

Our method was first assessed on simulated data in order to be independent of the input feature set and ground-truth issues. In (Gressin et al., 2013), we demonstrated that our methodology was not sensitive to various kinds of change, the ratio of change and the varying visual aspect of objects within a specific theme. In this paper, our method was applied on real 2D geographic DB and satellite images. One area of interest acquired with a satellite image is first described. Then, change detection results performed for two different DB are provided.

\subsection{Area of interest}

Our dataset covers a surface of $760 \mathrm{~km}^{2}$ in the Southern-West part of France. One acquisition of the Pleiades sensor in August 2012 is available. This acquisition is composed of two images: one panchromatic $0.5 \mathrm{~m}$ ortho-image, used to compute textural features and one four channels $2 \mathrm{~m}$ ortho-image, for spectral ones.

Three geographic DB are available on this area: the first one corresponds to the agricultural activities (crops, pastures, ..., called Field afterwards), the second one to forests (tree species, hedges etc.), and the third one to buildings. From these DB, two scenarios were generated. First, a DB with three very specific and detailed themes is proposed (called hereafter detailed DB): the Douglas fir closest forest class, the bread wheat class, and the building class. The two first themes represent two specific sub-themes of the two most populated themes of the area: Field and Forest. They were selected to assess the relevance of our method to the classification of "unknown" classes (compared to the building class that has been analysed through many approaches). Moreover, our method allows to not separate building class into several roof material dependent subclasses as it traditionally carried out. Change detection results on this DB are shown in Section 3.3. Secondly, a more global, inhomogeneous, and therefore challenging land-cover DB is generated (called global DB). All classes of the Field and Forest databases are merged into two themes, respectively. Therefore, the Field and Forest themes are composed of objects of varying visual aspects and sizes. Results on this DB are shown in Sec 3.4.

\subsection{Computed features}

First, spectral features were computed: they were derived from the red, green, blue and near-infrared channels available (e.g., NDVI, SAVI etc.), and were computed using the Orfeo-ToolBox library (Inglada and Christophe, 2009). Those twelve features are mainly useful to discriminate vegetation and water bodies. Then, one textural feature, namely the entropy of the histogram of gradient directions, was introduced in order to bring information about the structure of the texture around each pixel vicinity. It allows to best discriminate irregularly textured objects (such as forests) from featureless objects (as fields) (Trias-Sanz, 2006).Finally, dense SIFT descriptors are computed, in a regular pixel grid of the image (every 4 pixels), with a Gaussian filter of $\sigma=1.2$ (van de Sande et al., 2010) which is the default value of the algorithm. Then, a Principal Component Analysis is performed so as to extract the ten (empirical value) eigenvectors associated to the ten highest eigenvalues, so as to keep only the most discriminative information.

\subsection{Detailed DB results}

Classification results for three specific themes are discussed here. Figures $2 \mathrm{a}$ and $2 \mathrm{~b}$ focus on two areas of interest. These three examples allow to assess the performance of the training set selection and the classification steps. Since no ground-truth exists on this area, no quantitative study has been performed. However, a qualitative study was carried out and satisfactory classification results are obtained. One can notice that the three different themes are well labelled. Douglas fir is correctly discriminated from the other forest types, even though some confusion remains in shadow areas. Therefore, our method is able to deal with new objects and disappearing ones (Figures $2 \mathrm{c}$ and $2 \mathrm{~d}$ ). As for the Douglas fir theme, the bread wheat theme is also correctly discriminated from other fields (Figures $2 \mathrm{e}$ and $\mathrm{f}$ ). A partly disappeared object introduced some confusion, but did not prevent the 


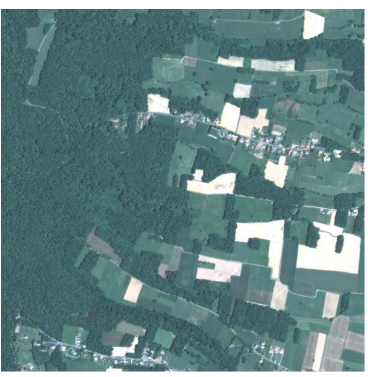

(a) AOI 1

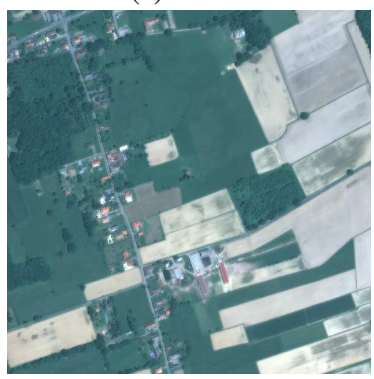

(b) $\mathrm{AOI} 2$

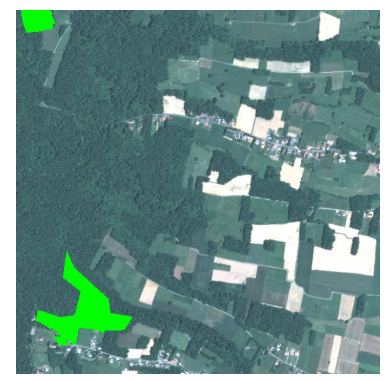

(c) Douglas fir DB (AOI 1)

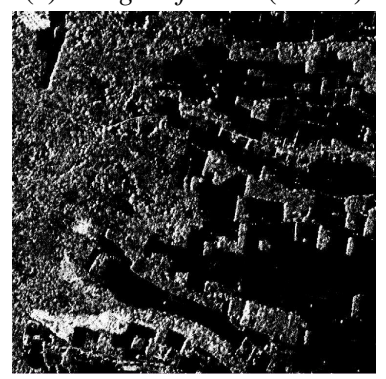

(d) Douglas fir results

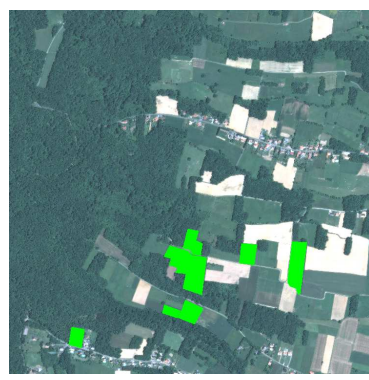

(e) Bread wheat DB (AOI 1)

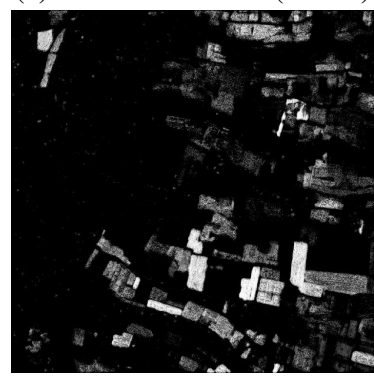

(f) Bread wheat results

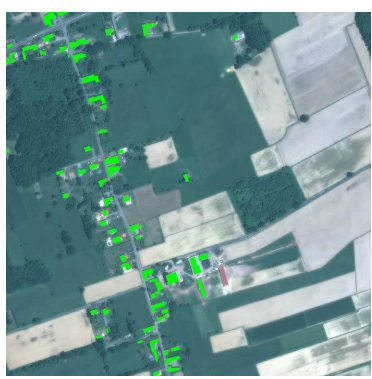

(g) Building DB (AOI 2)

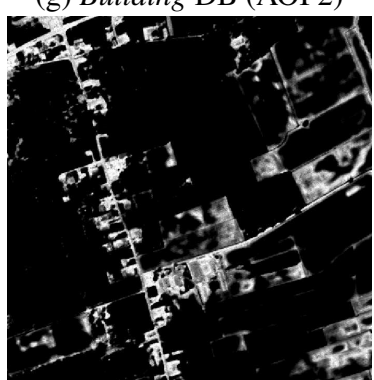

(h) Building results

Probability to belong to the current theme: 0

Figure 2: (a) and (b) The two specific areas of interest (AOI 1 and 2) of the Pléiades image. DB and the probability result at theme level for: (c) and (d) the douglas fir theme (AOI 1), (e) and (f) the bread wheat theme (AOI 1), (g) and (h) the building theme (AOI 2). No DB fusion has been performed here.

method from founding new objects. Finally, the classification for the building theme is the most confusing. Indeed, our method is able to detect almost all existing and new buildings. However, the classification fails to discriminate building from road, park lots, and dry parts of some fields, due to a restricted feature set (Figures $2 \mathrm{~g}$ and $\mathrm{h}$ ).

These results do not take into account the DB fusion process, that would allow to reduce confusion between the existing themes of the DB.

3.4 Global DB result

Finally, our change detection method was applied on the global $D B$ scenario. This DB is composed of two themes, namely Field and Forest. The Forest theme is composed of 50 objects of variable sizes from 5 different forest themes, and the Field theme gathers 190 objects from 20 different themes. Figure 3a and 3e show the area of interest and the final probability change map, respectively. One can notice extended red areas, corresponding to real changes on the ground, here new fields as well as forest disappearance or decrease. White areas are confusing areas, mainly objects that are not in the initial DB, like building, roads or other anthropic surfaces. These issues may be easily solved by introducing more themes in our initial DB.

Changes can also be studied theme by theme. Thus, Figure 3 shows the Forest theme (b), the classification result after fusion at the DB level (c), and the differences between the DB and the classification (d). This change map enhances new areas (in blue) and disappeared areas (in red). Most disappearance correspond to clearings, not included in the DB. Blue areas are new forest areas, extended forest areas, as well as hedges and copses that are again not in the DB. Indeed, only objects larger that a minimal size are included in the DB. If necessary, the knowledge of such specification would allow us to filter such objects.

Moreover, Figure 3 also shows for the Field DB (f), the classification result after fusion at the DB level (g), and the differences between both of them (h). This map shows many new fields (labelled in blue) as well as removed ones (in red). Many field borders are labelled as disappeared. This is due to the coarse delineation of Field objects during the initial image photointerpretation process.
Finally, similarly to the Detailed $D B$ scenario, no ground-truth is available and no qualitative assessment was possible. However, visual inspection showed satisfactory results, allowing to correctly detect the main changes between the DB and the image.

\section{CONCLUSION}

In this paper, we proposed a classification-based method that allowed to obtain satisfactory change maps between unknown 2D land-cover databases and a more recent satellite image. Our hierarchical method was based on a carefully designed per-object training set selection and a generic decision fusion, guided by the database layout. As this process is independent of both the database specifications and the input image, it can be applied on a large number of real cases. Our classification and per-theme fusion processes were first assessed on three specific and challenging themes. Despite a restricted number of features, our training set selection allowed to deal with object disappearance, and correctly found new objects. Then, the change detection methodology was applied on one land cover database composed of two very general themes (Field and Forest). We managed to find most changed areas. However, our method failed to discriminate some objects (as building from roads, or parking). Consequently, in future works, improvements of the method will focus on (1) the introduction of a large number of features, coupled with a feature selection step at the theme level and (2) a final regularization step at the DB level of the classifications obtained at the theme level.

\section{References}

Bovolo, F., Bruzzone, L. and King, R., 2013. Introduction to the special issue on analysis of multitemporal remote sensing data. IEEE TGRS 51(4), pp. 1867 - 1869.

Campedel, M., Moulines, E. and Datcu, M., 2005. Feature selection for satellite image indexing. ESA-EUSC: Image Information Mining, Frascati Italy.

Champion, N., Boldo, D., Pierrot-Deseilligny, M. and Stamon, G., 2010. 2D building change detection from high resolution satelliteimagery: A two-step hierarchical method based on 3D invariant primitives. PRL 31(10), pp. 1138 - 1147. 


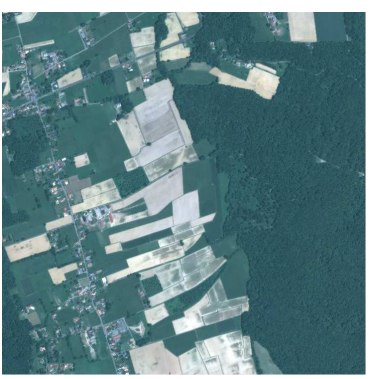

(a) Area of interest

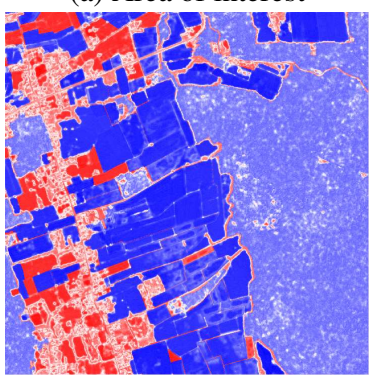

(b) Probability change map

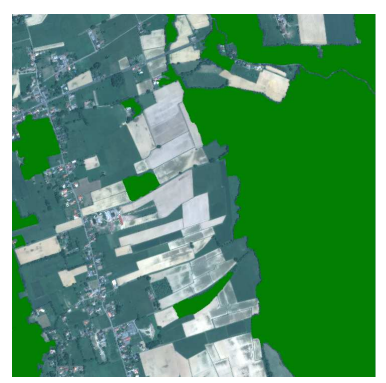

(c) Forest DB

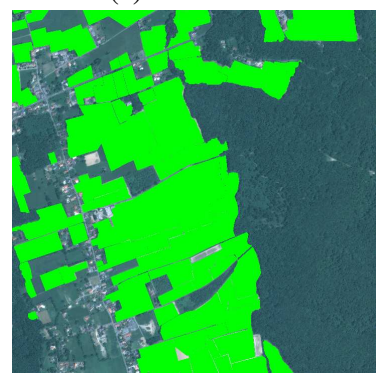

(d) Field DB

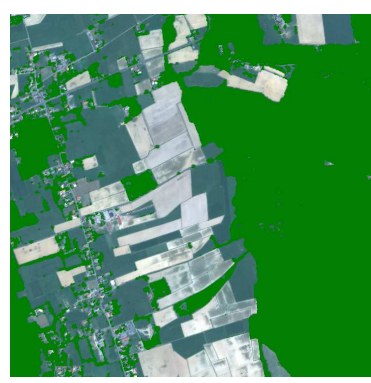

(e) Forest classification

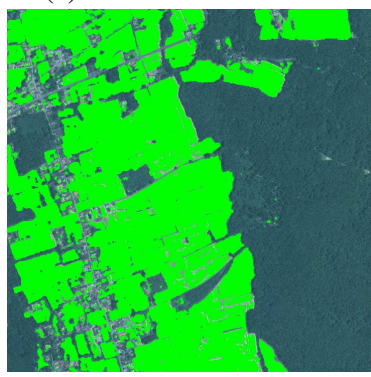

(f) Field classification

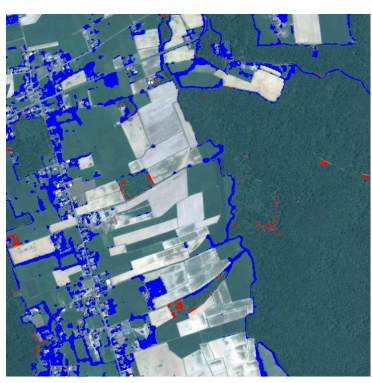

(g) Forest difference

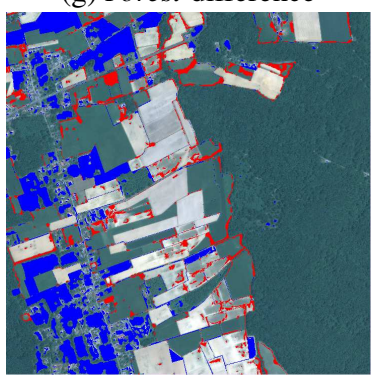

(h) Field difference

Figure 3: Results of the change detection method: (a) Orthoimage of the area of interest, (b) Probability change map (red: change - blue area: no change - white: confusing areas; (c) Forest theme (d) Field theme; (e) and (f) Classification results after DB fusion for the Forest and the Field themes, respectively; (g) and (h) Differences between the DB and the classification ( $\mathbf{\square}$ : new areas and $\mathbf{\square}$ disappeared areas) for the Forest and the Field themes, respectively.

Foody, G. M., Mathur, A., Sanchez-Hernandez, C. and Boyd, D. S., 2006. Training set size requirements for the classification of a specific class. RSE 104(1), pp. 1-14.

Gianinetto, M., 2008. Updating large scale topographic databases in Italian Urban areas with submeter QuickBird Images. IJNO 28(4), pp. 299-310.

Goyette, N., Jodoin, P., Porikli, F., Konrad, J. and Ishwar, P., 2012. Changedetection.net: A new change detection benchmark dataset. In: Computer Vision and Pattern Recognition Workshops (CVPRW).

Gressin, A., Vincent, N. and Paparoditis, N., 2013. Semantic approach in image change detection. In: ACIVS.

Helmholz, P., Becker, C., Breitkopf, U., Buschenfeld, T., Busch, A., Braun, C., Grunreich, D., Muller, S., Ostermann, J. and Pahl, M., 2012. Semi-automatic Quality Control of Topographic Data Sets. PE \& RS 78(9), pp. 959-972.

Holland, D., Boyd, D. and Marshall, P., 2006. Updating topographic mapping in Great Britain using imagery from highresolution satellite sensors. ISPRS Journal of Photogrammetry and Remote Sensing 60(3), pp. 212-223.

Hussain, M., Chen, D., Cheng, A., Wei, H. and Stanley, D., 2013. Change detection from remotely sensed images: From pixel-based to object-based approaches. ISPRS Journal of Photogrammetry and Remote Sensing 80, pp. 91-106.

Inglada, J. and Christophe, E., 2009. The Orfeo Toolbox remote sensing image processing software. In: IGARSS, pp. 733-736.

Lefebvre, A., Corpetti, T. and Hubert-Moy, L., 2008. Objectoriented approach and texture analysis for change detection in very high resolution images. In: IGARSS, pp. 663-666.

Lienou, M. and Campedel, M., 2009. Image semantic coding using OTB. In: IGARSS, pp. 745-748.

Marçal, A., Borges, J., Gomes, J. and Pinto Da Costa, J., 2005. Land cover update by supervised classification of segmented ASTER images. IJRS 26(7), pp. 1347-1362.
Miller, O., Pikaz, A. and Averbuch, A., 2005. Objects based change detection in a pair of gray-level images. Pattern Recognition 38(11), pp. 1976-1992.

Nemmour, H. and Chibani, Y., 2006. Multiple support vector machines for land cover change detection: An application for mapping urban extensions. ISPRS Journal of Photogrammetry and Remote Sensing 61(2), pp. 125-133.

Petitjean, F., Inglada, J. and Gancarski, P., 2012. Satellite Image Time Series Analysis Under Time Warping. IEEE TGRS 50(8), pp. 3081-3095.

Poulain, V., Inglada, J., Spigai, M., Tourneret, J.-Y. and Marthon, P., 2009. Fusion of high resolution optical and SAR images with vector data bases for change detection. In: IGARSS, pp. 956-959.

Radke, R., Andra, S., Al-Kofahi, O. and Roysam, B., 2005. Image change detection algorithms: a systematic survey. IEEE Transactions on Image Processing 14(3), pp. 294-307.

Robin, A., Moisan, L. and Le Hégarat-Mascle, S., 2010. An acontrario approach for subpixel change detection in satellite imagery. IEEE PAMI 32(11), pp. 1977-93.

Tokarczyk, P., Montoya, J. and Schindler, K., 2012. An evaluation of feature learning methods for high resolution image classification. ISPRS Annals of the Photogrammetry, Remote Sensing and Spatial Information Sciences I-3, pp. 389-394.

Trias-Sanz, R., 2006. Texture Orientation and Period Estimator for Discriminating Between Forests, Orchards, Vineyards, and Tilled Fields. IEEE TGRS 44(10), pp. 2755-2760.

van de Sande, K., Gevers, T. and Snoek, C., 2010. Evaluating color descriptors for object and scene recognition. IEEE PAMI 32(9), pp. 1582-96.

Walter, V., 2004. Object-based classification of remote sensing data for change detection. ISPRS Journal of Photogrammetry and Remote Sensing 58(3-4), pp. 225-238.

Wu, T., Lin, C. and Weng, R., 2004. Probability estimates for multi-class classification by pairwise coupling. JMLR 5, pp. 975-1005. 